\title{
Psychophysiological Effects of Massage-Myofascial Release After Exercise: A Randomized Sham-Control Study
}

\author{
Manuel Arroyo-Morales, M.D., Ph.D., ${ }^{1}$ Nicolas Olea, M.D., Ph.D., ${ }^{2}$ Marin Manuel Martínez, Ph.D., ${ }^{3}$ \\ Amparo Hidalgo-Lozano, M.S., ${ }^{1}$ Concepción Ruiz-Rodríguez, Ph.D., ${ }^{4}$ and Lourdes Díaz-Rodríguez, Ph.D. ${ }^{4}$
}

\begin{abstract}
Objective: The aim of this study was to evaluate the effect of massage on neuromuscular recruitment, mood state, and mechanical nociceptive threshold (MNT) after high-intensity exercise.

Design: This was a prospective randomized clinical trial using between-groups design.

Setting: The study was conducted at a university-based sports medicine clinic.

Participants: Sixty-two (62) healthy active students age 18-26 participated.

Interventions: Participants, randomized into two groups, performed three 30-second Wingate tests and immediately received whole-body massage-myofascial induction or placebo (sham ultrasound/magnetotherapy) treatment. The duration (40 minutes), position, and therapist were the same for both treatments.

Main outcome measures: Dependent variables were surface electromyography (sEMG) of quadriceps, profile of mood states (POMS) and mechanical nociceptive threshold (MNT) of trapezius and masseter muscles. These data were assessed at baseline and after exercise and recovery periods.

Results: Generalized estimating equations models were performed on dependent variables to assess differences between groups. Significant differences were found in effects of treatment on sEMG of Vastus Medialis (VM) $(p=0.02)$ and vigor subscale $(p=0.04)$. After the recovery period, there was a significant decrease in electromyographic (EMG) activity of VM $(p=0.02)$ in the myofascial-release group versus a nonsignificant increase in the placebo group $(p=0.32)$, and a decrease in vigor $(p<0.01)$ in the massage group versus no change in the placebo group $(p=0.86)$.

Conclusions: Massage reduces EMG amplitude and vigor when applied as a passive recovery technique after a high-intensity exercise protocol. Massage may induce a transient loss of muscle strength or a change in the muscle fiber tension-length relationship, influenced by alterations of muscle function and a psychological state of relaxation.
\end{abstract}

\section{Introduction}

$\mathbf{M}$ assage therapy is widely requested by sportspeople and trainers, but research findings on the benefits of its routine use have been controversial. ${ }^{1,2}$ Better control over study conditions is required to elucidate the effects of this activity in the sports setting and allow definitive conclusions to be drawn. ${ }^{3}$

Massage is commonly applied to sportspeople during periods of fatigue in training. Fatigue is associated with muscle fiber changes that reflect the increased effort required to maintain a given level of mechanical performance. ${ }^{4}$ These myoelectric alterations are observed as reductions in the amplitude of motor unit action potential ${ }^{5}$ and motor unit recruitment threshold.6,7 An increased electromyographic (EMG) amplitude ${ }^{8}$ and alteration of temporal muscle sequencing ${ }^{9}$ have been reported by authors using an eccentric exercise-induced delayed-onset muscle soreness (DOMS) model. However, supramaximal exercise (Wingate 30-s test) was found to produce a decrease in mean power frequency spectrum but no change in EMG amplitude. ${ }^{10,11}$ These EMG changes are related to the accumulation of metabolites in the periphery and an ineffective afferent command to the central nervous system to implement neural recruitment strategies.

\footnotetext{
${ }^{1}$ Department of Physical Therapy, University of Granada, Granada, Spain.

${ }^{2}$ Laboratorio Investigaciones Cientificas, Hospital Clinico Universitario San Cecilio Granada, Spain.

${ }^{3}$ Department of Physical Education, University of Granada, Granada, Spain.

${ }^{4}$ Department of Nursing, University of Granada, Granada, Spain.
} 
The relaxation produced by massage therapy has proved capable of reducing local fatigue rate $^{3}$ and muscular excitability $^{12-14}$ by inducing relaxation. However, the effectiveness of this therapy at the myoelectric level has not yet been established, ${ }^{2,15}$ and no evidence has been published on the EMG effects of myofascial release massage. Changes in neural recruitment after myofascial release can be studied by EMG recordings.

High-intensity training or competition is associated with mental fatigue and a generalized worsening of the state of mind. ${ }^{16-19}$ Post-exercise massage can generate well-being, a sense of calm, a reduction in anxiety, and an improvement in mood and perceived relaxation and recovery. ${ }^{1}$ Some authors consider that these benefits are solely related to psychological effects. ${ }^{13,20,21}$ This issue has been explored in the sports setting by studies using the Profile of Mood States (POMS). ${ }^{1,22}$

One of the most widespread theoretical models on the impact of massage focuses on its analgesic effects. ${ }^{21}$ Experience of pain is subjective and difficult to measure, ${ }^{23}$ and varied results have been obtained on the analgesic effect of post-exercise massage. ${ }^{20,24-26}$ Algometry pressure measurements have been successfully used to assess mechanical nociceptive thresholds (MNTs) in studies on post-exercise recovery. ${ }^{27-30}$ We could find no published studies on the mechanical nociceptive threshold (MNT) after myofascial release in the setting of post-exercise recovery. Single applications of different modalities of massage therapy have been reported to reduce various body/mind parameters, such as state anxiety, blood pressure, and heart rate, but not negative mood, immediate assessment of pain, or cortisol level. ${ }^{21}$ Deterioration of mood state has been associated with motor performance. $^{31}$

With this background, the present study was designed to follow the short-term effects of myofascial release on mind/ body parameters. Its objective was to investigate the shortterm effects of myofascial release as a recovery method after high-intensity exercise in healthy active subjects by monitoring their neuromuscular activity (surface electromyography activity [sEMG] of quadriceps), MNT, and mood state.

\section{Methods}

\section{Subjects}

Healthy active volunteers from the Sports and Exercise School and Health Sciences School of the University of Granada were enrolled in this study from October 2005 to March 2006. Inclusion criteria were 5-10 h/week of physical activity, no pharmaceutical drug intake in past 3 months, non-use of tobacco or other addictive substances, no signs / symptoms of disease, and no contraindication of highintensity exercise. Sixty-eight participants were randomly se-

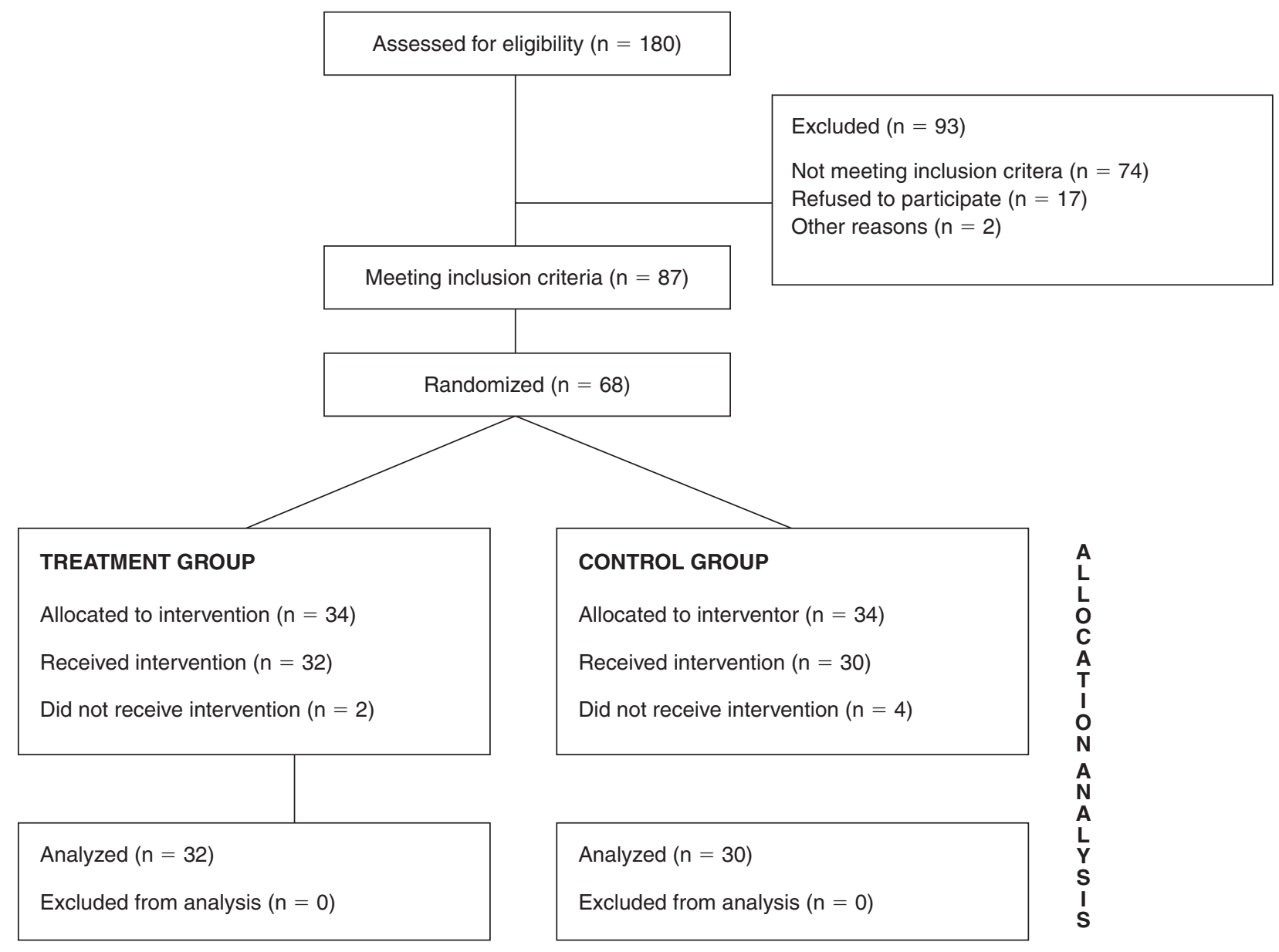

FIG. 1. Flow of participants 
lected for inclusion after estimating the required sample size by using a previously reported method. ${ }^{32}$ Written informed consent was obtained from all participants in this investigation, which was approved by the Ethics Committee of the University of Granada. The flow of participants is shown in Figure 1. The final study sample comprised 62 individuals, 37 men and 25 women, with a mean (SD) age of 21.1 (2.16) years, weight of $67.5(1.4) \mathrm{kg}$, height of $174.3(8.8) \mathrm{cm}$, body mass index (BMI) of 22.3 (1.4), and body fat percentage of 15.6 (5.4). Massage was part of the lifestyle of $34 \%$ of participants, was infrequently used by $58 \%$ of them, and had never been experienced by $8 \%$ of them.

\section{Experimental design}

A randomized sham-controlled study was performed using a between-group design. Subjects were assigned to massage or sham treatment by an envelope randomization method, matching the groups (treatment and sham) for sex. Volunteers entered the laboratory at the same time of day on three occasions, with a one-month interval between the first two sessions. All sessions took place between 5 p.m. and 9 p.m. At the first session, individuals underwent a medical examination (anthropometric study based on the Yuhasz-Carter skinfold method and a resting electrocardiogram [ECG]) and were screened for inclusion criteria. Familiarization was completed at the second session to ensure that all subjects knew the protocol and could complete the tasks required (see below), and to establish a reference performance value for comparison with the value obtained at the third (test) session. Following the procedure adopted in a previous study, ${ }^{3}$ the dietary intake (food and fluid) of subjects was recorded for the two days before the familiarization visit, and this intake was exactly replicated by the subjects during the two days before the final laboratory visit. Participants were instructed not to exercise heavily during the $24 \mathrm{~h}$ before the test. At the third session, baseline EMG, POMS, and MNT measurements were obtained after a 10-min rest in supine position. Subjects then performed a standardized light warm-up protocol, followed by three 30-s Wingate tests on an ergometer cycle (Monark ${ }^{\mathrm{TM}}$ 834, Varberg, Sweden) according to a previously published protocol. ${ }^{33}$ Wingate tests were separated by 3-min recovery periods. Each exercise test was performed against a braking force of $4.41 \mathrm{~J} \cdot$ pedal revolution ${ }^{-1} \cdot \mathrm{kg}$ body weight ${ }^{-1}$. For safety reasons and to improve exercise tolerance, the 3min recovery periods were divided between an initial active recovery phase (pedaling at a controlled $50 \mathrm{~W}$ work rate for $90 \mathrm{~s}$ ) and a subsequent passive recovery phase (90 s quiet sitting). After the third Wingate test, there was a 15-min period of active and passive recovery (10-min active/5-min passive). Subjects were encouraged to give their maximum performance during the exercise protocol and were offered an incentive (lottery ticket) if they improved on their performance in the familiarization (second) session. Electromyographic, POMS, and MNT measurements were obtained upon completion of the exercise protocol.

Immediately after the above determinations, subjects underwent sham or massage treatment. Disconnected ultrasound and magnetotherapy equipment was used for the 40min sham treatment, whereas the massage consisted of a whole-body myofascial release treatment lasting around 40 min (according to the tissue response observed) using the Barnes \& Upledger approach ${ }^{34,35}$ (Table 1). The aim of this massage approach was to influence the deleterious psychological and physiological effects of the exercise protocol used. The position of the patient and the areas treated were identical in the two protocols. All massages were administered by the same chartered physiotherapist using conventional oil (sweet almond oil, Acofarma) when necessary. Electromyogram, POMS, and MNT measurements were obtained immediately after the intervention.

\section{sEMG procedure}

Surface electromyography activity was used to quantify muscle activation of the vastus medialis (VM), vastus lateralis (VL), and rectus femoris (RF) muscles. Biometrics EMG hardware and software (Gwent, UK) were used. Electromyography signals were obtained by means of a Datalink EMG sensor SX320 (Biometrics) and were analyzed with Datalink version 3.0 software (Biometrics). Data were highpass filtered at $60 \mathrm{~Hz}$. Parameters were as follows: bandwidth 15-450 Hz, input impedance $2 \mathrm{M} \Omega$ (differential), common mode rejection ratio $92 \mathrm{~dB}$, maximum input voltage \pm $3 \mathrm{~V}$, sampling rate $1,000 \mathrm{~Hz}$ and gain 1,000 . The EMG procedure was as previously reported. ${ }^{36}$ Subjects performed one weight-bearing isometric maximal voluntary contraction (MVC) with their dominant limb (uniplanar knee extension). Three 5-s trials of each exercise were performed, separated by 2-min rest periods. Electromyography data for each muscle were integrated and the maximum root mean square (RMS) activity over a 0.5-s window was calculated for each trial. Data were not normalized because all comparisons made in this study were within-day.

\section{MNT procedure}

The MNT was measured by means of a pressure algometer (Wagner Instruments FPI 10). The tip of the algometer

Table 1. Massage-Myofascial Induction Protocol

\begin{tabular}{llr}
\hline Massage technique & \multicolumn{1}{c}{ Body area } & $\begin{array}{c}\text { Approximate time } \\
\text { (minutes) }\end{array}$ \\
\hline Long J-stroke & Gastrocnemius & 3 \\
Long J-stroke & Biceps femoris & 3 \\
Cross hand technique & Thoracolumbar fascia & 12 \\
Sustained pressure & Occipital condyles & 5 \\
V spread & Frontalis & 5 \\
Ear pull & Temporalis & 4 \\
Cross hand technique & Quadriceps & 8 \\
\hline
\end{tabular}


was applied perpendicular to the muscle, maintaining an application rate of $1 \mathrm{~kg} / \mathrm{s}^{-1}$. Subjects were instructed to make a signal immediately they experienced pain in order to establish their pain threshold.

The tip of the algometer was applied at the insertion of the superficial portion over the jaw angle (superficial masseter muscle) and the front-edge middle part of the upper trapezius muscle. These muscles are especially susceptible to increased contraction in response to different types of stress. ${ }^{37,38}$ These points on the muscles were marked with a yellow label to ensure that exactly the same sites were always used. The same professional operated the calibrated algometer in all cases, using the same rate of pressure for each measurement.

\section{POMS}

The Profile of Mood States questionnaire (Spanish version software) ${ }^{39}$ consists of 65 items on mood state. Scores (on a five-point scale of $0-4$ ) are grouped into six subscales: Tension-Anxiety, Depression-Dejection, Anger-Hostility, Vigor, Fatigue, and Confusion. Subscale scores were converted into T-scores for the statistical analysis, and the overall mood disturbance was also calculated.

\section{Statistical analysis}

A repeated-measures design was used with two fixed effect factors (treatment group and time) and a random effect factor (participants) nested in group. The classic analysis of this repeated-measures design was not indicated because the sphericity hypothesis was not met in many cases; therefore a generalized estimating equations (GEE) model was used. ${ }^{40}$ The model included two main effects (group and time) and the interaction of these effects. We could find no published studies on this topic, to avoid losing any relevant effects, we considered the interaction to be significant at $p<0.10$. If the interaction was not significant, we performed a main effects comparison of exercise to baseline with application of the Bonferroni correction. When the interaction was significant, comparisons were made between groups at each time point and between time points for each group, using the Bonferroni correction. When necessary, data were log-transformed to achieve homogeneity of variance. In the comparisons, $p<$ 0.05 was regarded as significant. The STATA 9.1 package (Stata Corp., College Station, TX) was used for the statistical analysis.

\section{Results}

\section{sEMG}

After the exercise protocol, the two groups showed a significant increase in the EMG amplitude of VM $(p<0.001)$, VL $(p<0.01$, and RF $(p<0.05)$ (Table 2$)$. Generalized estimating equations model analysis showed a significant effect of group $\mathrm{x}$ time interaction on VM $(p=0.02)$. The EMG of VM did not significantly differ between groups at baseline $(p=0.22)$. The EMG of VM was significantly lower versus baseline after massage treatment $(p=0.02)$ but not after sham treatment $(p=0.32)$. The EMG of VL $(p=0.20)$ and RF $(p=0.33)$ did not significantly differ between groups at baseline. Recovery changes in VL and RF were not analyzed because no significant effects of their interaction were observed in the GEE model analysis.

\section{POMS}

Generalized estimating equations model analysis showed significant effects of group $\times$ time on vigor $(p=0.04)$ and confusion $(p<0.10)$ (Table 3$)$. There was no significant difference in vigor between groups at baseline $(p=0.75)$, and both groups showed a significant reduction in vigor after the exercise protocol $(p<0.001)$. Vigor was significantly lower than at baseline $(p \leq 0.01)$ after the recovery with massage but similar to the baseline value after the sham treatment $(p=0.86)$. There was no significant difference between groups in confusion $(p=0.93)$, and a significant increase in confusion was observed after exercise protocol in both sham $(p=0.04)$ and treatment $(p<0.001)$ groups. After the re-

Table 2. Comparison of sEMG between Groups at Different Study Time Points ${ }^{a}$

\begin{tabular}{lccc}
\hline & $\begin{array}{c}\text { Sham group } \\
(\mathrm{n}=30)\end{array}$ & $\begin{array}{c}\text { Massage group } \\
(\mathrm{n}=32)\end{array}$ & p-value $^{*}$ \\
\hline Vastus medialis $(\mathrm{mV})$ & & & \\
$\quad$ Baseline & $290.8 \pm 185.7$ & $349.5 \pm 198.9$ & 0.02 \\
Exercise & $399.5^{* *} \pm 181.4$ & $482.5^{* *} \pm 293.4$ & \\
Recovery & $318.0 \pm 194.8$ & $293.6^{+} \pm 180.3$ & \\
Vastus lateralis (mV) & $171.6 \pm 149.1$ & $213.0 \pm 186.3$ & \\
Baseline & $210.4^{* *} \pm 156.5$ & $266.3^{* *} \pm 131.8$ & \\
Exercise & $201.6 \pm 97.2$ & $226.6 \pm 105.7$ & \\
$\quad$ Recovery & $158.9 \pm 96.5$ & $190.2 \pm 152.7$ & \\
Rectus femoris $(\mathrm{mV})$ & $178.3^{* *} \pm 87.1$ & $258.1^{* *} \pm 191.2$ & \\
Baseline & $186.6 \pm 146.4$ & $228.4 \pm 143.5$ & \\
Exercise & & & \\
Recovery & & & \\
\hline
\end{tabular}

aData are expressed as geometric mean \pm standard deviation. The values at different time points were tested by generalized estimating equations (GEE).

${ }^{*} p$-value for interaction effect between group and time by GEE analysis.

${ }^{* *} p<0.05$ between baseline and exercise measurements ${ }^{\dagger} P<0.05$ for within-group comparisons to baseline (for variables with significant interaction), using the Bonferroni correction.

$\mathrm{sEMG}=$ surface electromyography activity $(\mathrm{mV})$. 
Table 3. Comparison of Profile of Mood State (POMS) and Algometry of Masseter for Groups at Different Moments During Studya

\begin{tabular}{|c|c|c|c|}
\hline & Sham group & Massage group & $\mathrm{p}$ \\
\hline \multicolumn{4}{|l|}{ Tension } \\
\hline Baseline & $43.3 \pm 8.5$ & $43.3 \pm 7.9$ & \multirow{3}{*}{0.21} \\
\hline Exercise & $40.9 \pm 7.1$ & $42.5 \pm 7.3$ & \\
\hline Recovery & $32.9 \pm 3.8$ & $30.8 \pm 3.8$ & \\
\hline \multicolumn{4}{|l|}{ Depression } \\
\hline Baseline & $45.9 \pm 7.9$ & $44.3 \pm 7.3$ & \multirow{3}{*}{0.61} \\
\hline Exercise & $45.7 \pm 8.9$ & $45.4 \pm 7.2$ & \\
\hline Recovery & $40.8 \pm 4.9$ & $40.9 \pm 3.9$ & \\
\hline \multicolumn{4}{|l|}{ Anger } \\
\hline Baseline & $50 \pm 10.6$ & $47.2 \pm 9.3$ & \multirow{3}{*}{0.64} \\
\hline Exercise & $47.4 \pm 9.3$ & $46.9 \pm 9.1$ & \\
\hline Recovery & $42.3 \pm 4.7$ & $40.2 \pm 3.7$ & \\
\hline \multicolumn{4}{|l|}{ Vigor } \\
\hline Baseline & $54.6 \pm 8.7$ & $55.2 \pm 6.2$ & \multirow{3}{*}{$0.04^{*}$} \\
\hline Exercise & $47.7^{* *} \pm 7.1$ & $42.8^{* *} \pm 7.6$ & \\
\hline Recovery & $54.3 \pm 7.2$ & $50.3^{* * * *} \pm 7.0$ & \\
\hline \multicolumn{4}{|l|}{ Fatigue } \\
\hline Baseline & $43.0 \pm 6.7$ & $44.0 \pm 7.9$ & \multirow{3}{*}{0.15} \\
\hline Exercise & $56.6^{* *} \pm 9.5$ & $60.9^{* *} \pm 8.0$ & \\
\hline Recovery & $43.0 \pm 5.8$ & $42.5 \pm 7.3$ & \\
\hline \multicolumn{4}{|l|}{ Confusion } \\
\hline Baseline & $36.9 \pm 7.5$ & $37.0 \pm 7.0$ & \multirow{3}{*}{$0.10^{*}$} \\
\hline Exercise & $39.8^{* *} \pm 8.6$ & $43.6^{* *} \pm 8.1$ & \\
\hline Recovery & $32.7^{* *, *} \pm 4.1$ & $34.3 \pm 4.1$ & \\
\hline \multicolumn{4}{|c|}{ Mood disturbance } \\
\hline Baseline & $16433.3 \pm 3657.5$ & $16034.4 \pm 4092.7$ & \multirow{3}{*}{0.23} \\
\hline Exercise & $18350.0 \pm 4008.8$ & $19653.1 \pm 3566.1$ & \\
\hline Recovery & $13740.0 \pm 1782.7$ & $13834.4 \pm 1887.9$ & \\
\hline \multicolumn{4}{|c|}{ Masseter $\left(\mathrm{Kg} / \mathrm{cm}^{2}\right)$} \\
\hline Baseline & $2.1 \pm 0.6$ & $2.1 \pm 0.7$ & \multirow{3}{*}{0.20} \\
\hline Exercise & $1.9^{* *} \pm 0.6$ & $1.9^{* *} \pm 0.6$ & \\
\hline Recovery & $2.1 \pm 0.5$ & $2.2 \pm 0.6$ & \\
\hline \multicolumn{4}{|c|}{ Upper trepezius $\left(\mathrm{Kg} / \mathrm{cm}^{2}\right)$} \\
\hline Baseline & $2.5 \pm 0.8$ & $2.6 \pm 0.9$ & \multirow{3}{*}{0.60} \\
\hline Exercise & $2.3^{* *} \pm 0.9$ & $2.3^{* *} \pm 0.7$ & \\
\hline Recovery & $2.5 \pm 0.8$ & $2.7 \pm 0.9$ & \\
\hline
\end{tabular}

${ }^{a}$ Data are expressed as mean \pm standard deviation. The values at different moments were tested by generalized estimating equations. ${ }^{*} p<0.10,{ }^{* *} p<0.05$, respective baseline measurements.

covery strategy, confusion showed a significant decrease versus baseline $(p<0.01)$ in the sham group, whereas there was no significant difference versus baseline $(p=0.05)$ in the massage group (Table 3).

\section{MNT}

According to the GEE analysis, massage had no significant effect on MNT in the masseter $(p=0.20)$ or trapezius $(p=0.60)$ muscles. In both groups, MNT in the trapezius and masseter muscles significantly decreased $(p<0.001)$ after the exercise protocol but was similar $(p=0.30)$ to the baseline value at the end of the recovery intervention (Table 3 ).

No gender or age differences were observed in the massage or sham treatment effects studied (data not shown).

\section{Discussion}

This study offers the first demonstration that massage therapy, applied as a passive recovery strategy after intensive exercise, reduces the EMG amplitude of VM during maximum voluntary contraction, i.e., the maximum electric activity needed to perform this contraction. ${ }^{41}$ Electromyography amplitude has been associated with muscle strength $\mathrm{th}^{3,10}$ and with the relationship between muscle fiber tension and length, and it has been found that acute and chronic massage treatment can increase muscle fiber length (stretching). ${ }^{42}$ It is possible that, in our study, the muscle fiber length-tension relationship was modified by the massage treatment, reducing the EMG amplitude. A change in muscle fiber length with a transient loss of muscle strength may be related to a change in muscle architecture. ${ }^{10}$ It has recently been proposed that the mechanisms of myofascial release (transegrity, thixotropism) are based on changes to the architecture and functional state of the myofascial system. ${ }^{43} \mathrm{~A}$ parasympathetic vegetative response associated with massage-induced improvements in heart rate variability, blood pressure, ${ }^{32}$ and immune function ${ }^{44}$ may produce a local muscle response characterized by a decrease in motor unit action potential amplitude and motor unit firing rate.

The intermittent submaximal exercise protocol used in our study proved adequate to increase the EMG amplitude of 
VM, VL, and RF, compatible with local muscle fatigue. ${ }^{45,46} \mathrm{Re}-$ ports on EMG activity after a single Wingate test have been controversial, with one study describing a decrease ${ }^{47}$ and another maintenance ${ }^{10}$ of the amplitude of VM and VL. Our outcomes are consistent with previous findings of an increase in EMG amplitude after completion of a similar protocol, ${ }^{48}$ indicating that repeated Wingate tests can induce transient local muscle fatigue. The increased EMG amplitude detected in our study may be linked to physiologic events related to muscle fiber protection mechanisms in fatigue conditions. ${ }^{10,11}$

Following the same research line as other authors on the psychological effects of massage $e^{1,13}$ this study confirms a previous report ${ }^{20}$ that massage therapy does not induce positive mood state changes, at least in the short term, but reduces deleterious effects induced by high-intensity exercise. In the massage group, vigor decreased after exercise and then increased after recovery but to a level below the baseline value and the members of that group showed higher confusion levels than at baseline, unlike sham-treated subjects. Some authors associated a decrease in vigor and an increase in confusion with a reduction in sports performance. ${ }^{49,50}$ Taken together, these findings support previously held ideas ${ }^{51}$ that, to avoid any negative effects on performance, recovery massage protocols should not be applied before a competition. Lack of vigor and a tendency to confusion may indicate a subjective perception of the relaxation state generally induced by massage therapy. This state may be related to the ability of this type of manual therapy to restore balance to the autonomic nervous system after intermittent high-intensity exercise. ${ }^{32}$

In common with other authors, we found that massage has no effect on pain perception. , $24^{24}$ Likewise, our massage protocol does not appear to have any impact on the exercise-induced increase in MNT, which can spontaneously recover after a few minutes. ${ }^{52}$ We expected an increase in MNT to be associated with the relaxation induced by our massage protocol. ${ }^{32}$ New research on the MNT of muscles involved in exercise would be of interest to examine the relationships among psychological state, physiological state, and pain perception.

Study limitations include the absence of a direct evaluation of strength during the EMG recording. To assess the value of massage therapy for local recovery, future studies should explore whether the EMG decrease is associated or not with preservation of the strength developed during a maximal isometric contraction. A longer follow-up (e.g., 24-48 h) of massage-induced psychological changes would also be of interest to test the proposition that massage should be avoided in the two days before a competition. Strengths of the study are the good control and standardization measures adopted, overcoming some of the methodological limitations (control group conditions, sample size) of some previous studies on the effects of massage therapy.2,21 Our experience suggests the need for studies that use a withingroup design in order to reduce the variability in results.

In conclusion, the application of massage as a passive recovery strategy after high-intensity exercise reduces the EMG amplitude of VM during MVC and reduces vigor with respect to pre-exercise values.

\section{Acknowledgments}

The authors are grateful to the volunteers for their participation. The trial was funded by a research project grant (11/UPB10/06) from the Spanish Higher Sports Council.

\section{Disclosure Statement}

No competing financial interests exist.

\section{References}

1. Hemmings B, Smith M, Graydon J, Dyson R. Effects of massage on physiological restoration, perceived recovery, and repeated sports performance. Br J Sports Med 2000;34: 109-114.

2. Weerapong P, Hume PA, Kolt GS. The mechanisms of massage and effects on performance, muscle recovery and injury prevention. Sports Med 2005;35:235-256.

3. Robertson A, Watt JM, Galloway SD. Effects of leg massage on recovery from high intensity cycling exercise. Br J Sports Med 2004;38:173-176.

4. Orizio C. Muscle fatigue monitored by force, surface mechanomyogram and EMG. In: Nigg B, MacIsntosh BR, Mester J, eds. Biomechanics and Biology of Movement. Champaign, IL: Human Kinetics, 2000.

5. Patikas D, Michailidis C, Bassa H, Kotzamanidis C, et al. Electromyographic changes of agonist and antagonist calf muscles during maximum isometric induced fatigue. Int J Sports Med 2002:23:285-289.

6. Enoka RM, Stuart DG. Neurobiology of muscle fatigue. J Appl Physiol 1992;72:258-259.

7. Garland SJ, Enoka RM, Serrano LP, Robinson GA. Behaviour of motor units in human biceps brachii during a submaximal fatiguing contraction. J Appl Physiol 1994;76: 2411-2419.

8. Cheung K, Hume P, Maxwell L. Delayed onset muscle soreness: treatment strategies and performance factors. Sports Med 2003;33:145-164.

9. Zhou S, Carey MF, Snow RJ, Lawson DL, et al. Effects of muscle fatigue and temperature on electromechanical delay. Electromyogr Clin Neurophysiol 1998;38:67-73.

10. Hunter AM, St Clair Gibson A, Lambert MI, Nobbs L, et al. Effects of supramaximal exercise on the electromyographic signal. Br J Sports Med 2004;38:364-365.

11. Rana SR. Effect of the Wingate test on mechanomyography and electromyography. J Strength Cond Res 2006;20: 292-297.

12. Sullivan SJ, Williams LR, Seaborne DE, Morelli M. Effects of massage on alpha motoneuron excitability. Phys Ther 1991;71:555-560.

13. Cafarelli E, Flint F. The role of massage in preparation for and recovery from exercise. An overview. Sports Med 1992; 14:1-9.

14. Morelli M, Chapman CE, Sullivan SJ. Do cutaneous receptors contribute to the changes in the amplitude of the H-reflex during massage? Electromyogr Clin Neeurophysiol 1999;39:441-447.

15. Tanaka TH, Leisman G, Mori H, Nishijo K. The effect of massage on localized lumbar muscle fatigue. BMC Complement Altern Med 2002;14:2-9.

16. Raglin JS, Morgan WP, O'Connor PJ. Changes in mood states during training in female and male college swimmers. Int J Sports Med 1991;12:585-589.

17. Berglund B, Safstrom H. Psychological monitoring and modulation of training load of world-class canoeists. Med Sci Sports Exerc 1994;26:1036-1040.

18. Filaire E, Bernain X, Sagnol M, Lac G. Preliminary results on mood state, salivary testosterone:cortisol ratio and team performance in an professional soccer team. Eur J Appl Physiol 2001;86:179-184.

19. Mashiko T, Umeda T, Nakaji S, Sugawara K. Position related analysis of the appearance of an relationship between post- 
match physical and mental fatigue in university rugby football players. Br J Sports Med 2004;38:617-621.

20. Hilbert JE, Sforzo GA, Swensen T. The effects of massage on delayed onset muscle soreness. Br J Sports Med 2003; 37:72-75.

21. Moyer CA, Rounds J, Hannum JW. A meta-analysis of massage therapy research. Psychol Bull 2004;130:3-18.

22. Weinberg R, Jackson A, Kolodny K. (1988). The relationship of massage and exercise to mood enhancement. Sport Psychol 1988;2:202-211.

23. Kolt GS. Pain. In: Kolt GS, Snyder-Mackler L, Restrom P, eds. Physical Therapies in Sport and Exercise. London: Elsevier Science, 2003:129-144.

24. Weber MD, Servedio FJ, Woodall WR. The effects of three modalities on delayed onset muscle soreness. J Orthop Sports Phys Ther 1994;20:236-242.

25. Farr T, Nottle C, Nosaka K, Sacco P. The effects of therapeutic massage on delayed onset muscle soreness and muscle function following downhill walking. J Sci Med Sport 2002;5:297-306.

26. Lightfoot J, Char D, McDermot J. Immediate postexercise massage does not attenuate delayed onset of muscle soreness. J Strength Cond Res 1997;11:119-124.

27. Baker SJ, Kelly NM, Eston RG. Pressure pain tolerance at different sites on the quadriceps femoris prior to and following eccentric exercise. Eur J Pain 1997;1:229-233.

28. Johansson PH, Lindstrom L, Sundelin G, Lindstrom B. The effects of preexercise stretching on muscular soreness, tenderness and force loss following heavy eccentric exercise. Scand J Med Sci Sports 1999;9:219-225.

29. Persson AL, Hansson GA, Kalliomaki A, Mortiz U, et al. Pressure pain thresholds and electromyographically defined muscular fatigue induced by a muscular endurance test in normal women. Clin J Pain 2000;16:155-163.

30. Waling K, Sundenlin G, Ahlgren C, Jarvholm B. Perceived pain before and after three exercise programs-A controlled clinical trial of women with work-related trapezius myalgia. Pain 2000;85:201-207.

31. Kitaoka K, Ito R, Araki H, Sei H, et al. Effect of mood state on anticipatory postural adjustments. Neurosci Lett 2004; 370:65-68.

32. Arroyo-Morales M, Olea N, Martínez M, Moreno-Lorenzo $\mathrm{C}$, et al. Effects of myofascial release after high intensity exercise. A randomized clinical trial. J Manip Phys Ther 2008; 31:217-223.

33. Engels HJ, Fahlman MM, Wirth JC. Effects of ginseng on secretory IgA, performance and recovery from interval exercise. Med Sci Sports Exerc 2003;35:690-696.

34. Barnes JF. Myofascial Release: The Search for Excellence, a Comprehensive Evaluatory and Treatment Approach. Paoli, PA: Rehab Services, Inc. \& Myofascial Release Seminar: 1990.

35. Updleger JE, Vredevoogd J. Craniosacral Therapy. Seattle: Eastland Press, 1993.

36. Hertel J, Earl JE, Tsang KK, Miller SJ. Combining isometric knee extension exercises with hip adduction or abduction does not increase quadriceps EMG activity. Br J Sports Med 2003;38:210-213.

37. Nakamura K. Taoda K, Kitahara T, Tsujimura H, et al. Effect of mental stress on hemoglobin dynamics in trapezius muscles. San ei Shi 2007;49:225-233.
38. Hidaka O, Yanagi M, Takada K. Changes in masseteric hemodynamics time-related to mental stress. J Dent Res 2004;83:185-190.

39. Hernández Mendo A, Ramos Pollán. Herramienta 2: Informatiación del Profile of Mood Status de MacNair, Lorr y Dropleman. In: Hernández Mendo A, Ramos Pollán R, eds. Introducción a la informática aplicada a la psicología del deporte. Madrid: Ra-Ma, 1996.

40. Diggle PJ, Liang KZ, Zegen SL. Generalized estimating equations. In: Diggle PJ, Liang KZ, Zegen SL, eds. Analysis of Longitudinal Data. New York: Oxford University Press, 1994:146-167.

41. Kumar S, Narayan Y, Zedka M. An electromyographic study of unresisted trunk rotation with normal velocity among healthy subjects. Spine 1996;21:1500-1512.

42. Wiktorsson-Moller M, Oberg B, Ekstrand J, Gillquist J. Effects of warming up, massage, and stretching on range of motion and muscle strength in the lower extremity. Am J Sports Med 1983;11:249-252.

43. Schleip R, Klingler W, Lehmann-Horn F. Active fascial contractility: Fascia may be able to contract in a smooth muscle-like manner and thereby influence musculoskeletal dynamics. Med Hypotheses 2005;65:273-277.

44. Arroyo-Morales M, Olea N, Ruíz C, Luna del Castillo JD, et al. Massage after exercise. Responses of immunologic and endocrine markers: A randomized single-blind placebo-controlled study. J Strength Cond Res in press.

45. Habes DJ. Use of EMG in a kinesiological study in industry. Appl Ergon 1984;15:297-301.

46. Luttman A, Jager M, Sokeland J, Lauring W. Electromyographical study on surgeons in urology. Determination of muscular fatigue. Ergonomics 1996;39:285-313.

47. Vandewalle H, Maton B, Le Bozec S, Guerenbourg G. An electromyographic study of an all-out exercise on a cycle ergometer. Arch Int Physiol Biochim Biophys 1991;99:89-93.

48. Billaut F, Basset FA, Giocomoni M, Lemaitre F, et al. Effect of high-intensity intermittent cycling sprints on neuromuscular activity. Int J Sports Med 2006;27:25-30.

49. Cox RH. Mood state and athletic performance. In: Cox RH, ed. Sports Psychology: Concepts and Applications. New York: McGraw-Hill, 2002:177-190.

50. Lane AM, Terry PC, Beedie CJ, Curry DA, et al. Mood and performance: Test of a conceptual model with a focus on depressed mood. Psychology Sport Exerc 2001;2:157-172.

51. Holey E, Cook E. Massage in sport. In: Holey E, Cook E, eds. Evidence-Based Therapeutic Massage: A Practical Guide for Therapists. London: Elsevier 2003:295-308.

52. Petovaara A, Huapaniemi T, Virtanen A, Johansson G. The influence of exercise on dental pain threshold and the release of stress hormones. Physiol Behav 1984;33:923-926.

Address reprint requests to: Manuel Arroyo-Morales, M.D., Ph.D.

Department of Physical Therapy

University of Granada

Avenida Madrid s/n

18071 Grenada

Spain

E-mail: marroyo@ugr.es 